\title{
Trend of livestock population and nutritional evaluation of available feed resources in coastal areas of Noakhali district
}

\author{
MZ Rahman, M Ershaduzzaman, KS Huque, MY Ali*
}

Bangladesh Livestock Research Institute, Savar, Dhaka 1341, Bangladesh

\begin{abstract}
A survey work was undertaken with a total of $200 \mathrm{farm}$ households of the coastal areas of Noakhali were categorized into landless, marginal, small, medium and large based on their landholding and sheep population. All categories of farmers had higher number of sheep compared to goat and cattle. In case of lamb (0-4months) farmers didn't supplied concentrate. The average deficiency of DM ( $g / h / d), M E$ $(\mathrm{MJ} / \mathrm{h} / \mathrm{d})$ and $\mathrm{CP}(\mathrm{g} / \mathrm{h} / \mathrm{d})$ of lamb were $70.24,0.97$ and 13.35 , respectively. For growing sheep (4-15 month) small, medium and large farmers provided little but landless and marginal farmers provided very little amount of concentrate. The average deficiency of DM $(\mathrm{g} / \mathrm{h} / \mathrm{d}), \mathrm{ME}(\mathrm{MJ} / \mathrm{h} / \mathrm{d})$ and CP $(\mathrm{g} / \mathrm{h} / \mathrm{d})$ of growing sheep were 257.31, 2.62 and 34.75, respectively. In case of adult ewe (>15 month, milking) the average deficiency of $\operatorname{DM}(\mathrm{g} / \mathrm{h} / \mathrm{d}), \operatorname{ME}(\mathrm{MJ} / \mathrm{h} / \mathrm{d})$ and $\mathrm{CP}(\mathrm{g} / \mathrm{h} / \mathrm{d})$ were 441.87, 2.81 and 35.45, respectively. Livestock farming contributed about $17.2 \%$ of annual family income in which the contribution of sheep of the total household income was about $11 \%$ followed by goat (3.58\%) and cattle $(2.62 \%)$. The available feed resources were durba, asamilata, chaila, bothoua, gamma, alias grass, shon, bontil, khesari, pakisthani lata, bean leaf, ipil ipil, mayahagoni, kadam, boroi leaf, jackfruit, babla, mango leaf, kitchen wastes, rice straw, and rice bran, til oil cake. Surveyed information on existing available nutrient status of sheep and feed resources would help to develop a feeding system of sheep in coastal areas of Bangladesh.
\end{abstract}

Key words: Coastal, forages, lamb, nutrient

Bangladesh Animal Husbandry Association. All rights reserved. Bang. J. Anim. Sci. 2014.43 (3): $213-217$

\section{Introduction}

There are about 2.78 million sheep in Bangladesh (BER, 2008). Economically sheep are the important livestock species in this country. Native sheep are small (18-25 kg), highly prolific (2-3 lambs per lambing and two lambing per year) and meat producing $(7-10 \mathrm{~kg})$ animals (Dymundsson et al. 1972). Sheep rearing are practicing throughout the country, but higher concentration is found in the coastal region of Noakhali and some char lands of Cox's bazar. Development of feeding system enhances the productivity of small ruminants specially sheep (Fahumy et al., 1990). Sheep rearing are directly are involved with poverty alleviation, employment generation and nutrient supply. Native sheep are extremely resistant to infectious diseases including PPR (Haque et al. 1988). They could be reared under harsh management condition and relatively poor quality feed. Unlike goat they are nondestructive social animal with relatively easy management. Recently, Government of Bangladesh has taken an initiative to improve sheep production of the country to alleviate poverty of the poor people. Soon, it will be apparent that sheep could be an important meat producing animal of the country. Feed deficits in the country are considered as the major hindrance for Livestock production. Farmers usually provide feed to their small ruminants by letting them graze on fallow land, canals and roadside. Dietary nutrients usually energy and protein are the major important factors affecting meat production in sheep. Therefore, a survey work was undertaken to identify the existing feeding and management practices of sheep in selected coastal region and to evaluate the available feed resources at coastal areas. 


\section{Feeding systems of sheep in coastal areas}

\section{Materials and methods}

A total of 200 farm households of the village were categorized into landless, marginal, small, medium and large based on their landholding (BBS, 2005). To achieve the above objectives a survey was done from September 2011 to April 2012 through structured questionnaire. Existing feeding system of sheep was determined in some selected sheep populated coastal areas (Companygonj, Subarnachar and Kobirhat upazilla) of Noakhali. Year round nutrient availability in terms of energy and protein was estimated from the feed intake of sheep and their chemical composition (DM, CP, CF, EE and Ash) of feed. Existing nutrient availability was related to nutrient requirement of sheep and the gap was estimated. The available feed resource (natural pastures, browses, crops residues, kitchen wastes, concentrates etc) was identified and samples were transferred to research station for chemical analysis.

The samples were subjected to chemical analysis for the determination of dry matter (DM) and crude protein ( $C P$ ) following the methods of $A O A C$ (1995). The acid detergent fibre (ADF) was determined according to Goering and Van Soest (1970). All the samples were analyzed in duplicate and the mean values were recorded.

The collected data were compiled, tabulated and analyzed in accordance with the objectives following using SPSS-11.5 statistical program.

\section{Results and discussion}

Landless farm families were dominating (44\%), followed by marginal (23\%), small (21\%), medium (9\%) and large (3\%) (Table 1). Landless, marginal, small, medium and large farm families owned on an average $0.07,0.62$, $1.85,2.90$ and 7.90 acres of land including homestead, pond/ditch, cultivable land and fallow land, respectively. Among 88 landless farm families, 12 farmers (13.64\%) had no homestead land of their own. Again 68 (77\%) landless farmers possess no cultivable land at all. Number of livestock species in landless farm families was small. Livestock number gradually increased from landless to large farmers except goat. All categories of farmers had higher number of sheep compared to goat and cattle. Average number of sheep, goat and cattle were $6.42 \%$, $1.29 \%$ and $0.73 \%$, respectively.

The nutrient availability of sheep in different stages is shown in Table 2. Sheep was usually grazed surrounding areas of homestead, roadside and fallow land. In winter farmers transferred their sheep to the char areas near the sea for feeding. In rainy season there were a lot of green grasses and farmers collect it by cutting from different fields. Landless and marginal farmers regularly provided tree forages like jack fruit, mango and ipil ipil. Depending upon individual ability farmers provided concentrate.

Table 1. General information and livestock status of farm families (Mean $\pm S E$ )

\begin{tabular}{|c|c|c|c|c|c|c|}
\hline \multirow{2}{*}{ Parameters } & \multicolumn{5}{|c|}{ Farm category } & \multirow{2}{*}{ All farm } \\
\hline & Landless & Marginal & Small & Medium & Large & \\
\hline 1. Farm family (No.) & 88 & 46 & 42 & 18 & 6 & 200 \\
\hline$(n=200)$ & $(44 \%)$ & $(23 \%)$ & $(21 \%)$ & $(9 \%)$ & $(3 \%)$ & $(100 \%)$ \\
\hline 2. Land size (acre farm ${ }^{-1}$ ) & $0.07 \pm 0.36$ & $0.62 \pm 0.24$ & $1.85 \pm 0.14$ & $2.90 \pm 0.17$ & $7.90 \pm 0.19$ & $1.05 \pm 0.78$ \\
\hline i. Homestead & $0.03 \pm 0.10$ & $0.15 \pm 0.08$ & $0.25 \pm 0.20$ & $0.35 \pm 0.21$ & $0.50 \pm 0.10$ & $0.14 \pm 0.13$ \\
\hline ii. Pond/ditch & $0.01 \pm 0.09$ & $0.02 \pm 0.7$ & $0.10 \pm 0.08$ & $0.15 \pm 0.15$ & $0.20 \pm 0.08$ & $0.04 \pm 0.16$ \\
\hline iii. Cultivable land & $0.03 \pm 0.12$ & $0.40 \pm 0.12$ & $1.30 \pm 0.17$ & $2.10 \pm 0.16$ & $6.80 \pm 0.12$ & $0.77 \pm 0.14$ \\
\hline iv. Fallow land & - & $0.05 \pm 0.21$ & $0.20 \pm 0.18$ & $0.30 \pm 0.31$ & $0.40 \pm 0.15$ & $0.09 \pm 0.12$ \\
\hline \multicolumn{7}{|l|}{ 3. Possession of livestock } \\
\hline i. Sheep & $4.32 \pm 0.12$ & $6.21 \pm 0.09$ & $7.31 \pm 0.16$ & $11.21 \pm 0.06$ & $18.39 \pm 0.15$ & $6.42 \pm 0.98$ \\
\hline ii. Goat & $1.82 \pm 0.21$ & $1.63 \pm 0.09$ & $0.35 \pm 0.18$ & $0.15 \pm 0.08$ & $0.90 \pm 0.09$ & $1.29 \pm 0.77$ \\
\hline iii. Cattle & $0.15 \pm 0.11$ & $0.82 \pm 0.15$ & $1.11 \pm 0.14$ & $2.21 \pm 0.10$ & $2.00 \pm 0.13$ & $0.73 \pm 0.53$ \\
\hline
\end{tabular}

Figures in the parenthesis indicate percent of total 
In case of lamb $(0-4 \mathrm{~m})$ total DM supplied $(\mathrm{g} / \mathrm{h} / \mathrm{d})$ were $72.45,73.30,74.23,80.50$ and 82.32 of landless, marginal, small, medium and large farmers, respectively. Landless farmers didn't supply any concentrate. The average deficiency of DM $(\mathrm{g} / \mathrm{h} / \mathrm{d}), \operatorname{ME}(\mathrm{MJ} / \mathrm{h} / \mathrm{d})$ and $\mathrm{CP}(\mathrm{g} / \mathrm{h} / \mathrm{d})$ of lamb were $70.24(48.68 \%), 0.97(48.50 \%)$ and $13.35(62.86 \%)$, respectively (Table 2$)$. Nutrient deficiency was gradually decreased from landless to large farmers. The weight gain of suckling lambs is closely associated with level of nutrient intake. Weaning age of lambs is from 4 week to 5 months of age. Therefore, after the age of 2 weeks, milk should be offered less and adequate feed and roughage should be available to the lamb. Adequate feeds provoke the high intake and performance of sheep. Energy intake is the most important factor in production. Feed allowance, therefore, must be adequate. But the finding of nutrient availability was too low to requirement. The weight gain of suckling lambs is closely associated with the level of nutrient intake (Economides 1984).

Table 2. Existing feeding system of sheep in the surveyed areas

\begin{tabular}{|c|c|c|c|c|c|c|}
\hline Farm category & Landless & Marginal & Small & Medium & Large & All farm \\
\hline \multicolumn{7}{|l|}{ Lamb (0-4 months) } \\
\hline Grasses DM (g/h/d) & $48.33^{a} \pm 2.30$ & $51.24^{a} \pm 3.70$ & $60.12^{a b} \pm 4.3$ & $70.19^{b} \pm 4.60$ & $72.21^{b} \pm 3.80$ & $54.16 \pm 2.99$ \\
\hline Tree forage DM (g/h/d) & $24.12^{b} \pm 2.90$ & $22.13^{b} \pm 2.90$ & $14.11^{\mathrm{a}} \pm 3.3$ & $10.31^{\mathrm{a}} \pm 2.20$ & $10.11^{\mathrm{a}} \pm 1.97$ & $19.90 \pm 2.98$ \\
\hline Concentrate DM (g/d/h) & - & & - & - & - & - \\
\hline Total DM supply (g/h/d)) & $72.45^{a} \pm 3.30$ & $73.30^{a} \pm 4.4$ & $74.23^{\mathrm{ab}} \pm 2.9$ & $80.50^{b} \pm 5.20$ & $82.32^{b} \pm 4.88$ & $74.05 \pm 3.21$ \\
\hline Deficiency DM (g/h/d) & $\begin{array}{c}71.85^{b} \pm 3.90 \\
(49.79 \%)\end{array}$ & $\begin{array}{c}70.93^{b} \pm 3.9 \\
(48.15 \%)\end{array}$ & $\begin{array}{c}70.07^{b} \pm 5.3 \\
(49.55 \%)\end{array}$ & $\begin{array}{c}63.80^{\mathrm{a}} \pm 3.80 \\
(44.21 \%)\end{array}$ & $\begin{array}{c}61.98 \mathrm{a} \pm 5.11 \\
(43.95 \%)\end{array}$ & $\begin{array}{c}70.24 \pm 4.44 \\
(48.68 \%)\end{array}$ \\
\hline Deficiency ME(MJ/h/d) & $\begin{array}{c}0.99 \pm 0.56 \\
(49.48 \%)\end{array}$ & $\begin{array}{c}0.98 \pm 0.39 \\
(49.20 \%)\end{array}$ & $\begin{array}{c}0.97 \pm 0.43 \\
(48.45 \%)\end{array}$ & $\begin{array}{c}0.88 \pm 0.39 \\
(44.45 \%)\end{array}$ & $\begin{array}{c}0.86 \pm 0.31 \\
(43.00 \%)\end{array}$ & $\begin{array}{c}0.97 \pm 0.32 \\
(48.50 \%)\end{array}$ \\
\hline Deficiency CP(g/h/d) & $\begin{array}{c}13.58 \pm 1.21 \\
(63.94 \%)\end{array}$ & $\begin{array}{c}13.45 \pm 0.96 \\
(63.33 \%)\end{array}$ & $\begin{array}{c}13.31 \pm 1.12 \\
(62.67 \%)\end{array}$ & $\begin{array}{c}12.40 \pm 1.11 \\
(58.39 \%)\end{array}$ & $\begin{array}{c}12.13 \pm 0.87 \\
(57.11 \%)\end{array}$ & $\begin{array}{c}13.35 \pm 2.21 \\
(62.86 \%)\end{array}$ \\
\hline \multicolumn{7}{|l|}{ Growing (4-15 months) } \\
\hline Grasses DM (g/h/d) & $240.13^{a} \pm 5.21$ & $264.24 \mathrm{a} \pm 5.21$ & $288.39 \mathrm{~b} \pm 4.98$ & $278.41^{b} \pm 3.39$ & $312.32^{\mathrm{b}} \pm 5.54$ & $260.16 \pm 4.87$ \\
\hline Tree forage DM (g/h/d) & $36.12^{a} \pm 2.98$ & $24.19 \mathrm{~b} \pm 1.98$ & $26.40^{\mathrm{b}} \pm 2.22$ & $21.68^{b} \pm 2.98$ & $24.51^{\mathrm{b}} \pm 4.98$ & $29.68 \pm 2.10$ \\
\hline Concentrate DM (g/h/d) & $8.78^{a} \pm 1.65$ & $13.17 \mathrm{a} \pm 2.12$ & $35.12^{b} \pm 3.21$ & $52.62^{c} \pm 4.32$ & $61.46 c \pm 6.78$ & $20.33 \pm 4.21$ \\
\hline Total DM supply(g/h/d)) & $285.03^{a} \pm 6.43$ & $301.61^{a} \pm 6.12$ & $349.92^{b} \pm 4.32$ & $352.77^{b} \pm 3.51$ & $398.31^{c} \pm 5.74$ & $310.16 \pm 3.23$ \\
\hline $\begin{array}{l}\text { Deficiency DM } \\
(\mathrm{g} / \mathrm{h} / \mathrm{d})\end{array}$ & $\begin{array}{c}282.97^{c} \pm 6.33 \\
(49.82 \%)\end{array}$ & $\begin{array}{c}266.40 c \pm 5.65 \\
(46.90 \%)\end{array}$ & $\begin{array}{c}218.09 \mathrm{~b} \pm 6.34 \\
(38.40 \%)\end{array}$ & $\begin{array}{c}215.23^{b} \pm 4.36 \\
(37.90 \%)\end{array}$ & $\begin{array}{c}169.71 \mathrm{a} \pm 7.32 \\
(29.88 \%)\end{array}$ & $\begin{array}{c}257.31 \pm 2.53 \\
(45.30 \%)\end{array}$ \\
\hline $\begin{array}{l}\text { Deficiency ME } \\
(\mathrm{MJ} / \mathrm{h} / \mathrm{d})\end{array}$ & $\begin{array}{c}2.50^{b c} \pm 0.48 \\
(49.90 \%)\end{array}$ & $\begin{array}{c}2.36^{\mathrm{b}} \pm 1.11 \\
(47.12 \%)\end{array}$ & $\begin{array}{c}1.93^{\mathrm{ab}} \pm 0.87 \\
(38.52 \%)\end{array}$ & $\begin{array}{c}1.86^{\mathrm{a}} \pm 0.88 \\
(37.21 \%)\end{array}$ & $\begin{array}{c}1.50^{\mathrm{a} \pm 2.43} \\
(29.94 \%)\end{array}$ & $\begin{array}{c}2.62 \pm 3.21 \\
(49.29 \%)\end{array}$ \\
\hline Deficiency CP (g/h/d) & $\begin{array}{c}36.04 \pm 1.61 \\
(66.40 \%)\end{array}$ & $\begin{array}{c}35.30 \pm 1.38 \\
(65.04 \%)\end{array}$ & $\begin{array}{c}33.84 \pm 1.23 \\
(62.35 \%)\end{array}$ & $\begin{array}{c}31.11 \pm 1.52 \\
(58.79 \%)\end{array}$ & $\begin{array}{c}26.22^{\mathrm{a}} \pm 1.67 \\
(48.31 \%)\end{array}$ & $\begin{array}{c}34.75 \pm 2.97 \\
(64.02 \%)\end{array}$ \\
\hline \multicolumn{7}{|l|}{ Adult (>15 months) } \\
\hline Grasses DM (g/h/d)) & $288.31^{\mathrm{a}} \pm 4.43$ & $336.29 b \pm 5.22$ & $360.18^{c} \pm 6.81$ & $408.21^{d} \pm 5.60$ & $432.31^{d} \pm 3.65$ & $329.55 \pm 5.33$ \\
\hline Tree forage DM $(\mathrm{g} / \mathrm{h} / \mathrm{d})$ & $60.11^{b} \pm 4.92$ & $48.91^{\mathrm{a} \pm 3.34}$ & $45.60^{\mathrm{a}} \pm 6.11$ & $48.19^{a} \pm 4.98$ & $52.82^{a} \pm 4.71$ & $53.19 \pm 3.99$ \\
\hline Concentrate DM $(\mathrm{g} / \mathrm{h} / \mathrm{d})$ & $43.91^{\mathrm{a}} \pm 2.88$ & $52.68^{b} \pm 5.1$ & $52.68^{b} \pm 5.11$ & $61.46^{c} \pm 3.98$ & $79.02^{\mathrm{d}} \pm 4.66$ & $50.40 \pm 4.79$ \\
\hline Total DM supply $(\mathrm{g} / \mathrm{h} / \mathrm{d})$ & $392.33^{a} \pm 5.44$ & $437.88^{b} \pm 4.21$ & $458.38^{b} \pm 8.32$ & $517.86^{c} \pm 7.55$ & $564.15^{\mathrm{d}} \pm 3.87$ & $433.14 \pm 6.67$ \\
\hline $\begin{array}{l}\text { Deficiency DM } \\
(\mathrm{g} / \mathrm{h} / \mathrm{d})\end{array}$ & $\begin{array}{c}482.67^{d} \pm 6.70 \\
(55.16 \%)\end{array}$ & $\begin{array}{c}437.12^{c} \pm 5.47 \\
(49.95 \%)\end{array}$ & $\begin{array}{c}416.62^{c} \pm 4.08 \\
(47.61 \%)\end{array}$ & $\begin{array}{c}357.14^{\mathrm{b}} \pm 3.99 \\
(40.82 \%)\end{array}$ & $\begin{array}{c}310.85^{\mathrm{a}} \pm 3.21 \\
(35.53 \%)\end{array}$ & $\begin{array}{c}441.87 \pm 6.01 \\
(50.50 \%)\end{array}$ \\
\hline $\begin{array}{l}\text { Deficiency ME } \\
(\mathrm{MJ} / \mathrm{h} / \mathrm{d})\end{array}$ & $\begin{array}{c}3.06^{c} \pm 0.98 \\
(55.04 \%)\end{array}$ & $\begin{array}{c}2.78^{b} \pm 0.61 \\
(50 \%)\end{array}$ & $\begin{array}{c}2.64^{b} \pm 0.47 \\
(47.48 \%)\end{array}$ & $\begin{array}{c}2.26^{a} \pm 0.26 \\
(40.64 \%)\end{array}$ & $\begin{array}{c}1.98 \mathrm{a} \pm 0.19 \\
(36.61 \%)\end{array}$ & $\begin{array}{l}2.81 \pm 0.96 \\
(50.36 \%)\end{array}$ \\
\hline Deficiency CP(g/h/d) & $\begin{array}{c}36.71^{b} \pm 2.11 \\
(63.87 \%)\end{array}$ & $\begin{array}{c}36.19 \mathrm{~b} \pm 2.43 \\
(62.97 \%)\end{array}$ & $\begin{array}{c}34.50^{\mathrm{b}} \pm 1.98 \\
(60.03 \%)\end{array}$ & $\begin{array}{c}32.53^{b} \pm 2.75 \\
(56.60 \%)\end{array}$ & $\begin{array}{c}26.63 a \pm 1.69 \\
(46.33 \%)\end{array}$ & $\begin{array}{c}35.45 \pm 4.98 \\
(61.68 \%)\end{array}$ \\
\hline
\end{tabular}

Means with different superscript in the same row differed significantly $(p<0.05)$ 


\section{Feeding systems of sheep in coastal areas}

For growing sheep (4-15 months) total DM supplied $(\mathrm{g} / \mathrm{h} / \mathrm{d})$ were $285.03,301.61,349.92$, 352.77 and 398.31 of landless, marginal, small, medium and large farmers, respectively. Small, medium and large farmers provided little but landless and marginal farmers provided very little amount of concentrate. The ingredients of concentrates were wheat bran, rice polish and rice bran but large farmers provided til oil cake. The average deficiency of DM $(\mathrm{g} / \mathrm{h} / \mathrm{d})$, ME $(\mathrm{MJ} / \mathrm{h} / \mathrm{d})$ and $\mathrm{CP}(\mathrm{g} / \mathrm{h} / \mathrm{d})$ of growing sheep were $257.31(45.30 \%), 2.62(49.29 \%)$ and 34.75 $(64.02 \%)$, respectively (Table 2 ). Sheep were allowed for grazing during the day on natural pasture, homestead forest, fallow land. Sometimes, mother ewes with small lambs were kept tethered just besides the house. In terms of feeding management of livestock, most farmers practiced mixed management feeding systems. Inadequate feed supply resulted in low energy intake. The importance of adequate nitrogen intake in relation to energy intake for the performance of lambs (Egan 1969; Kempton and Leng 1980).

In case of adult ewe ( $>15$ months, milking) total DM intake $(\mathrm{g} / \mathrm{h} / \mathrm{d})$ were $392.33,437.88,458.38$, 517.86 and 564.15 of landless, marginal, small, medium and large farmers, respectively. The average deficiency of DM $(\mathrm{g} / \mathrm{h} / \mathrm{d}), \operatorname{ME}(\mathrm{MJ} / \mathrm{h} / \mathrm{d})$ and $\mathrm{CP}(\mathrm{g} / \mathrm{h} / \mathrm{d})$ were $441.87(50.5 \%), 2.81$
(50.36\%) and $35.45(61.68 \%)$, respectively. Findings indicate that nutrient availability of sheep were very poor (Table 2). Inadequate nutrition, particularly of energy, depressed the reproductive performance of extensively (HFRO 1979) or intensively managed sheep (Orskov 1982). Sexual maturity of sheep is advanced by good feeding and the energy stimulates oestrus activity within the normal breeding season, ovulation rate, fertilization and survival of ova and the maintenance of the resultant embryos to term as viable lambs (Gunn 1967). Low levels of energy during late pregnancy lead to pregnancy toxaemia (Economides and Louca 1981). The productivity of sheep in developing countries is generally low, mainly because of under feeding and poor management (1980). Productivity is also low in highlands because of low quality feed intake (HFRO 1979).

Annual household income sources were broadly comes from crops, homestead base enterprise (gardening, nonfarm activities), business, service and livestock (Table 3). Livestock farming contributed about $17.2 \%$ of total annual family income. However, the contribution of sheep of the total household income was about $11 \%$ share followed by goat $(3.58 \%)$ and cattle $(2.62 \%)$.

Table 3. Household income of farm families (Tk./h/y)

\begin{tabular}{|c|c|c|c|c|c|c|}
\hline \multirow{2}{*}{ Sources of income } & \multicolumn{5}{|c|}{ Farm category } & \multirow{2}{*}{ All farm } \\
\hline & Landless & Marginal & Small & Medium & Large & \\
\hline Crops & $1190.11 \pm 11.21$ & $11823.59 \pm 38.87$ & $22943.39 \pm 44.21$ & $44878.48 \pm 53.11$ & $88903.52 \pm 89.44$ & $14767.35 \pm 45.22$ \\
\hline Homestead base enterprise & $570.29 \pm 9.11$ & $980.30 \pm 18.76$ & $1150.30 \pm 21.11$ & $1380.88 \pm 16.56$ & $1560.77 \pm 19.41$ & $908.35 \pm 21.21$ \\
\hline Business & $25075.10 \pm 22.12$ & $31541.22 \pm 18.54$ & $22930.39 \pm 23.34$ & $30803.49 \pm 43.11$ & $35300.56 \pm 50.11$ & $26934.23 \pm 33.21$ \\
\hline Service & $12557.45 \pm 38.77$ & $28330.11 \pm 46.1$ & $38990.69 \pm 45.3$ & $44380.81 \pm 52.21$ & $22300.62 \pm 48.23$ & $24892.53 \pm 39.66$ \\
\hline Livestock: & $44185.25 \pm 28.33$ & $87491.00 \pm 33.21$ & $101398.74 \pm 27.65$ & $146409.53 \pm 45.11$ & $183762.63 \pm 51.11$ & $81763.77 \pm 36.24$ \\
\hline i. Sheep & $6174.69 \pm 19.32$ & $8861.82 \pm 35.21$ & $10448.37 \pm 36.61$ & $16008.46 \pm 38.11$ & $26285.31 \pm 41.22$ & $9190.57 \pm 29.31$ \\
\hline ii. Goat & $3023.87 \pm 15.66$ & $2708.19 \pm 15.88$ & $581.51 \pm 9.33$ & $249.22 \pm 32.5 .11$ & $1495.32 \pm 8.33$ & $2141.63 \pm 11.11$ \\
\hline iii. cattle & $593.74 \pm 9.44$ & $3245.77 \pm 15.66$ & $4354.09 \pm 12.11$ & $8708.19 \pm 18.77$ & $7916.53 \pm 19.78$ & $2929.11 \pm 16.44$ \\
\hline Contribution of sheep (\%) & $12.56^{c} \pm 0.95$ & $10.13^{b} \pm 1.11$ & $10.31^{c} \pm 1.32$ & $10.93^{c} \pm 1.54$ & $14.31^{c} \pm 2.15$ & $11.00 \pm 2.22$ \\
\hline Contribution of goat (\%) & $6.15^{b} \pm .098$ & $3.10 a \pm 0.76$ & $0.57 a \pm 0.01$ & $0.17 \mathrm{a} \pm 0.01$ & $0.81^{a} \pm 0.0$ & $3.58 \pm 0.64$ \\
\hline Contribution of cattle (\%) & $1.21^{a} \pm 0.03$ & $3.71^{a} \pm 0.09$ & $4.29 b \pm 0.14$ & $5.95^{b} \pm 012$ & $4.31^{b} \pm 0.15$ & $2.62 \pm 0.08$ \\
\hline Total (\%) & $19.92 \pm 0.17$ & $16.94 \pm 0.15$ & $15.17 \pm 0.12$ & $17.05 \pm 0.91$ & $19.43 \pm 1.54$ & $17.20 \pm 1.11$ \\
\hline
\end{tabular}

Means with different superscript in the same row differed significantly $(p<0.01)$ 
Locally available forages are shown in Table 4. Feed resources were durba, asamilata, chaila, bothoua, gamma, alias grass, shon, bontil, khesari, pakistani lata, bean leaf, ipil ipil, mayahagoni, kadam, boroi leaf, jackfruit, babla, mango leaf, kitchen wastes, rice straw, rice bran and til oil cake (Table 4). Grasses were available in April to October except pakisthani lata round the year and khesari in November to March. Tree forages and concentrates were available round the year. All of these feed resources were good source of $\mathrm{CP}$ and ME.

Table 4. Nutrient composition (\%) of feed ingredients

\begin{tabular}{lccccc}
\hline \multirow{2}{*}{$\begin{array}{l}\text { Available } \\
\text { forages }\end{array}$} & $\mathrm{DM}$ & \multicolumn{3}{c}{ DM basis (\%) } & ME (MJ/ \\
\cline { 3 - 4 } & & $\mathrm{CP}$ & $\mathrm{ADF}$ & Ash & $\mathrm{kg} \mathrm{DM})$ \\
\hline Durba grass & 32.44 & 8.51 & 35.54 & 14.11 & 7.11 \\
Chaila grass & 24.97 & 6.48 & 30.32 & 9.5 & 8.98 \\
Khesari grass & 22.76 & 20.53 & 28.43 & 12.43 & 9.56 \\
Alias grass & 20.23 & 7.21 & 32.34 & 10.36 & 6.68 \\
Shon grass & 21.21 & 6.80 & 36.32 & 10.56 & 6.80 \\
Bontil grass & 23.36 & 7.43 & 31.78 & 12.13 & 7.80 \\
Pakisthani lata & 26.54 & 7.80 & 32.23 & 13.12 & 8.20 \\
Bean & 28.32 & 19.65 & 35.32 & 13.56 & 7.32 \\
Boroi & 32.44 & 11.76 & 34.23 & 8.98 & 6.98 \\
Kadam & 29.78 & 16.89 & 33.45 & 10.32 & 10.98 \\
Ipil ipil & 28.44 & 24.22 & 34.32 & 10.21 & 12.20 \\
Mango & 44.62 & 6.87 & 38.11 & 9.6 & 4.44 \\
Babla & 27.23 & 19.21 & 32.54 & 6.80 & 8.87 \\
Jack fruit & 28.32 & 11.87 & 44.23 & 11.56 & 6.8 \\
\hline
\end{tabular}

\section{Conclusion}

Survey findings indicate that farmers prefer sheep farming than goat or cattle. Sheep farming contributed a significant role of total annual family income. But deficiencies of nutrient are the major constraints for livestock rearing. Surveyed information about existing feeding system and nutrient composition of feedstuffs would help to develop a feeding system for livestock in coastal areas of Bangladesh.

\section{References}

AOAC (1995). Official Methods of Analysis. (16 ${ }^{\text {th }}$ edition), Association of Official Analytical Chemist. Washington DC.

BBS (Bangladesh Bureau of Statistics) (2005). Year Book of Agricultural Statistics of Bangladesh. Planning Division Ministry
Planning, Government of Peoples Republic of Bangladesh, Shere-e-bangla Nagar, Dhaka. P. 226.

BER (Bangladesh Economic Review) (2008). Government of The people's republic of Bangladesh, Finance Division, Ministry of Finance.

Devendra C (1980). Potential of sheep and goats in less developed countries. Journal of Animal Science 51, 461 - 473.

Dymundsson OR, JL Lee (1972). Attainment of puberty and reproductive performance in clun Forest ewe lambs. J. Agric. Sci. Camb., 78: 39- 45.

Economides S (1984). FAO European Cooperative Research Subnetwork meeting on Goat Production. 16-18, October, 1984. Grangeneuve, Switzerland.

Economides S, Louca A (1981). Nutrition and Systems of goat feeding, International Symposium, Tours-France France.

Egan AF (1969). Small ruminant production in the developing countries. 16: 451-462.

Fahumy MH (1990). Reproductive performance, growth and wool production of Romanov sheep in Canada. Small Ruminant Research 2: 253-264.

Goering HG and Van Soest PJ (1970). Forage fiber analysis. ARD. USDA. Washington, DC. Agriculture Handbook, no. 379, 20pp.

Gunn RG (1967). Health management of ewes during pregnancy, HFRO 4th Report, P. 5758.

Haque A, CS Ali, N Ahmad HA Samad, ZI Qureshi (1988). Studies on the reproductive efficiency in Kajli sheep. Sarhad Journal of Agriculture, 4: 241-7.

HFRO (Hill Farming Research Organization) (1979). Science and Hill Farming, Published by HFRO. Edinburgh.

Kempton TJ, Leng RA (1980). Glucose metabolism in growing lambs. Australian Journal of Biological Science, British J ournal of Nutrition, 42: 289-302.

Orskov ER (1982). Sheep and Goat Production. (Ed. IE Coop). Elsevier Scientific Publishing Co. Amsterdam-Oxford-New York. 